\title{
Children with poorly controlled asthma: Randomized controlled trial of a home-based environmental control intervention
}

\author{
Arlene Manns Butz, ScD, RN, CRNP ${ }^{1,2}$, Mary E. Bollinger, DO ${ }^{3}$, Jean Ogborn, MD, MPH ${ }^{4}$, \\ Tricia Morphew, $\mathbf{M S}^{5}$, Shawna S. Mudd, DNP, CRNP2 ${ }^{2}$ Joan E. Kub, PhD, RN ${ }^{6,7}$, Melissa H. \\ Bellin, PhD, LCSW ${ }^{8}$, Cassia Lewis-Land, MS ${ }^{1}$, Kelli DePriest, BSN ${ }^{2}$, and Mona Tsoukleris, \\ PharmD, MS $^{9}$
}

${ }^{1}$ Division of General Pediatrics and Adolescent Medicine, Baltimore, Maryland ${ }^{2}$ The Johns Hopkins University School of Nursing, University of Maryland, Baltimore, Maryland ${ }^{3}$ School of Medicine, Department of Pediatrics, Baltimore, Maryland ${ }^{4}$ Department of Pediatric Emergency Medicine, Johns Hopkins University, Baltimore, Maryland ${ }^{5}$ Morphew Consulting LLC, Bothell, Washington ${ }^{6}$ Department of Nursing, USC Suzanne Dworak-Peck School of Social Work, Los Angeles, California ${ }^{7}$ University of Maryland, Baltimore, Maryland ${ }^{8}$ School of Social Work, Johns Hopkins University School of Medicine, Baltimore, Maryland ${ }^{9}$ The School of Pharmacy, Johns Hopkins University School of Medicine, Baltimore, Maryland

\section{Abstract}

Background: Few trials have tested targeted environmental control (EC) interventions based on biomarkers of second hand smoke (SHS) exposure and allergen sensitization in reducing asthma emergency department (ED) visits in children with poorly controlled asthma.

Methods: Overall, 222 children with poorly controlled asthma were randomized into a homebased EC intervention (INT) or control (CON) group and followed for ED visits over 12 months. All children received allergen-specific IgE serologic testing and SHS exposure biomarker testing to inform the EC intervention. Pharmacy data was examined for asthma medication fills. Cox proportional hazards and multivariate regression models were performed to examine factors associated with repeat ED visits.

Results: There was no difference in increased risk of $>1$ ED visit at 12 months between INT and CON groups. Most children (75\%) had moderate/severe persistent asthma. Over half (56\%) had SHS exposure and $83 \%$ tested positive for $>1$ allergen sensitization. Among children without SHS exposure, the median time to first recurrent ED visit differed by group (CON: 195; INT: >365 days) after adjusting for child age, allergic sensitization, medication fills prior to baseline, controller medication use, and the interaction between group status and SHS exposure. Children who had positive allergic sensitizations, younger, had increased controller medication use and randomized to the CON group and had no SHS exposure had increased risk for a repeat ED visit over 12 months.

Correspondence: Arlene M. Butz, ScD, RN, CRNP, Department of Pediatrics, Johns Hopkins University School of Medicine, 200 N. Wolfe St, Baltimore 21287, MD, USA. abutz@jhmi.edu. 
Conclusions: In this study, a home-based EC intervention was not successful in reducing asthma ED revisits in children with poorly controlled asthma with SHS exposure. Allergic sensitization, young age, and increased controller medication use were important predictors of asthma ED visits.

\section{Keywords}

allergic sensitization; asthma; emergency department; environmental control; second hand smoke (SHS) exposure

\section{1 | INTRODUCTION}

Asthma affects seven million children in the US with the annual healthcare expenditures at $\$ 56$ billion. ${ }^{1,2}$ This high cost is primarily due to high asthma related emergency department (ED) and hospitalization rates. Although one out of five US children with asthma receive ED treatment, ${ }^{2} \mathrm{ED}$ utilization for asthma care is disproportionately higher among minority, and low income children ${ }^{3}$ with 12 -month revisit rates ranging from $6 \%$ to $34 \%$ for inner-city children. ${ }^{4,5}$ These revisits are costly, anxiety provoking, and largely preventable. ${ }^{6,7}$

Discerning the risk factors associated with increased asthma ED revisits is complex since risks include adverse environmental exposures, child sociodemo-graphic and health characteristics, and caregiver asthma management practices and beliefs. ${ }^{8} 9$ Asthma ED revisits have been associated with exposures to indoor allergens, respiratory infections, and second hand smoke (SHS) ${ }^{10-14}$; controller medication underuse and short acting beta agonist (SABA) overuse, ${ }^{7} 15-18$ improper medication delivery device technique, parental worry about asthma medication use and younger child age. $9,19,20$

Prior intervention studies to prevent risk for poor asthma outcomes mainly focused on reduction in environmental exposure to specific allergens. ${ }^{21-23}$ Exposure and allergen sensitization to mouse, cockroach and dust mite allergen detected in inner-city children are associated with increased risk factor for poorly controlled asthma. ${ }^{22-26}$ Home-based asthma education and environmental control (EC) programs have the potential to reduce asthma morbidity and lower ED utilization, ${ }^{21,27}$ yet prior environmental interventions have demonstrated equivocal results in effectively reducing indoor allergens. ${ }^{22,23}$ Single allergen interventions, school-based and low-intensity programs are often ineffective due to the multiple environmental exposures and complex management issues experienced by inner city children. ${ }^{27-29}$ Alternatively, multifaceted home-based EC interventions addressing common indoor allergens and SHS exposures are likely to be effective especially when biomarkers confirm sensitization to indoor allergens and cotinine for SHS exposure, but few prior EC interventions target indoor allergens and SHS exposures. One multifaceted homebased intervention to reduce wheezing symptoms in young children targeted indoor allergen and tobacco smoke exposures while also addressing maternal asthma management (Childhood Asthma Prevention Study, CAPS). ${ }^{8}$ Despite significant reduction in home cockroach allergen and SHS exposure, there was no decrease in wheezing symptoms, ED visits, or hospitalizations. ${ }^{8}$ 
The objective of this study was to determine the efficacy of a home-based EC intervention, based on biomarkers of allergen sensitization and cotinine concentrations for SHS exposure, to reduce recurrent or repeat asthma ED visits among inner-city children with persistent asthma. Secondary analysis examined other co-factors associated with the risk of ED revisits and intervention effects on symptom free days.

\section{2| METHODS}

\subsection{Design and study setting}

This prospective randomized controlled trial tested the efficacy of an ED and home-based EC intervention among inner-city children with frequent asthma ED visits. Children aged 312 years with persistent asthma were recruited and enrolled during an asthma ED visit from August 2013 through February 2016. Inclusion criteria were physician diagnosed persistent asthma, having two or more ED asthma visits or $>1$ hospitalization over the past 12 months and residing in the Baltimore metropolitan area. Exclusion criteria included foster care and significant other non-asthma respiratory conditions. After written informed caregiver consent and child verbal assent (child age $>8$ years) were obtained, children were randomly assigned using random-number generation prepared by biostatistician and stratified by child age (3-5 vs 6-12 years) to ensure groups were balanced by age, into the intervention (INT), or attention control $(\mathrm{CON})$ group via opaque sealed envelopes viewed by the study coordinator. Research staff collecting the data were blinded to group assignment. As shown in Figure 1, of the 554 child/caregivers screened for study enrollment, 117 children were ineligible for enrollment, and another 215 caregivers declined to participate resulting in randomization of 222 children into the study. During the enrollment ED visit, all children received allergen-specific IgE serologic testing measured by fluorescent enzyme immunoassay (FEIA) to identify allergen sensitization to ten common environmental allergens and salivary cotinine measurement to screen for exposure to SHS. Concurrently, caregivers were asked about the child's indoor exposure to mice, cockroaches, cats, and dogs. Using REDCap $\odot$ application ${ }^{30}$ surveys were administered to the child's caregiver at the baseline, 6 and 12 month time points in the ED or participants' homes by trained research assistants. The Johns Hopkins Medical Institutional and the University of Maryland Institutional Review Boards approved the study and the study was registered with https:// ClinicalTrials.gov, (https://www.clinicaltrials.gov): NCT01981564.

\subsection{Interventions}

2.2.1 Asthma express intervention-Intervention (INT) was a home-based asthma follow-up and environmental control (EC) educational program delivered by trained nurses and nurse practitioners (NP) to the child and caregiver (Table 1). INT children received a medical follow-up visit in the ED within 7 days of the enrollment asthma ED visit to evaluate: (i) asthma symptoms; (ii) guideline-based controller and rescue medication use and inhaler technique; (iii) review allergen and cotinine lab results using graphs to represent positive allergen sensitizations and child's level of cotinine. Additionally, INT children received two home nurse visits for targeted EC education and remediation based on the child's allergen sensitization results (eg, positive mouse allergy: set up mouse traps; positive cockroach allergy: set up cockroach bait and delivered kitchen trash cans). A nurse trained in 
integrated pest management conducted all home visits. For caregivers of children with positive cotinine (>1.0 ng/mL) results, brief motivational interviewing sessions were conducted to implement a total home smoking ban. Last, primary care provider (PCP) asthma care within 4 weeks was scheduled for the child.

2.2.2 Control-The attention control $(\mathrm{CON})$ group of caregivers and children received three home nurse visits over 3 months to provide (i) standard asthma education regarding guideline-based controller and rescue medication use; (ii) evaluate inhaler technique; (iii) basic EC education without home remediation; and (iv) assistance with scheduling PCP follow-up asthma care (Table 1). Child allergen and cotinine test results were mailed to CON caregivers and child's PCP; the home nurse reviewed the allergen and cotinine results with the caregiver. No active home remediation was conducted. Referrals to smoking cessation programs were provided to caregivers who had a smoker residing in the home. No motivational interviewing sessions were conducted. Fidelity checks were performed with INT and CON nurses using home visit checklists that logged behavioral and educational aspects of each home visit. No unintended effects occurred in either group.

\section{3 | Outcomes}

2.3.1 Asthma healthcare utilization-The primary outcome was having $>1 \mathrm{ED}$ repeat asthma visit over the 12-month study period based on caregiver report and medical record review at 12 months. Other healthcare utilization measures included number of PCP visits for non-urgent asthma care, prior ICU asthma admissions, asthma specialty care visits over the past 2 years and type of health insurance reported at baseline. Number of ED visits, ICU admissions and type of health insurance were verified by review of electronic medical record (EMR) with moderate to high agreement (ED visits: 82\%, ICU admissions: 81\%, and type of health insurance: $99 \%$ agreement).

\section{$2.4 \mid$ Covariates}

2.4.1 Asthma severity assessment-Baseline asthma severity was based on the National Asthma Education and Prevention Program (NAEPP) guideline for asthma severity ${ }^{31}$ and determined by caregiver report of: (i) number of symptom days over the past 14 days and symptom nights over the past 30 nights; (ii) short-acting $\beta_{2}$-agonist (SABA) use over past 14 days; (iii) activity limitation over the past 7 days; (iv) number of oral corticosteroid courses in the past year; and (v) number of asthma ED visit or hospitalizations over the past 12 months.

\subsubsection{Asthma medication use and caregiver worry about medication side}

effects-Pharmacy records of asthma medications dispensed over 12 months were obtained at baseline (prior 12 months) and at the 12-month follow-up (12 months post enrollment). A total of 24 months of pharmacy fill data were collected for each participant. Pharmacy records included the dispensing date, product name, strength, dosage form, and quantity dispensed. Rescue medication was defined as short acting $B$-agonists (SABA) and controller medications were defined as inhaled corticosteroids (ICS), leukotriene modifiers (LTM), and long acting beta agonists (LABA). Oral corticosteroid (OCS) prescription fills were analyzed separately. As a proxy of appropriate controller medication use, the asthma 
medication ratio $(\mathrm{AMR})^{32}$ or the total number controller medication canisters or equivalents divided by the total number of controller and SABA medication canisters or equivalents was calculated using 12-month pharmacy fill data. AMR ratios of 0.50 or higher have been associated with decreased asthma morbidity. ${ }^{32}$

Caregiver worry over side effects of asthma medication was recorded at baseline, 6 and 12 months, using a single item from the Pediatric Asthma Caregiver Quality of Life Questionnaire Scale ("How worried or concerned are you about your child's asthma medications and side effects?"). ${ }^{33}$ Responses were categorized into two groups: "very, fairly, or somewhat worried" versus "a little, hardly or not worried."

2.4.3 Serologic allergen specific IgE test-Serum specific IgE testing, collected during the baseline enrollment ED visit, was performed by a private commercial laboratory using the (ImmunoCap $\left.{ }^{\circledR}\right)$ fluorescent enzyme immunoassay (FEIA). Specific IgE to ten common environmental allergens were measured including mouse, cockroach, cat, dog, timothy grass, alternaria, and aspergillus molds, oak tree, common ragweed, and house dust mite. The degree of sensitization ranged from $<0.35$ to $>100 \mathrm{kU} / \mathrm{L}$ of specific IgE to any of the allergens tested. Results of $>0.35 \mathrm{kU} / \mathrm{L}$ were considered as an increased likelihood of presence of clinical allergy and thus for the purpose of this analysis were defined as "positive."

\subsubsection{Second hand smoke (SHS) exposure-Saliva samples for cotinine} measurement, a biomarker of recent nicotine exposure, were collected during the enrollment ED asthma visit, 6 and 12 months follow-up visits. Saliva was collected using a 3-cm cotton swab that was placed under the child's tongue for $1 \mathrm{~min}$ to absorb $1 \mathrm{~mL}$ of saliva (Salimetrics, State College, PA). The swab was placed in a $2 \mathrm{~mL}$ vial and stored at $-20^{\circ}$ Centigrade for transport to the lab, then centrifuged, and analyzed at the Johns Hopkins Institute for Clinical and Translational Research (ICTR) lab using Enzyme Immuno-assay (EIA) analysis. The lower limit of cotinine sensitivity was $0.05 \mathrm{ng} / \mathrm{mL}$ and average intra and inter-assay coefficients of variation were less than $5.8 \%$ and $7.9 \%$, respectively. Based on prior findings in comparable child population, positive SHS exposure was defined as a salivary cotinine level $>1.0 \mathrm{ng} / \mathrm{mL}^{34}$

\subsection{Analyses}

The primary outcome in this study was having $>1$ ED repeat asthma visit over the 12-month follow-up period. Assuming 90\% power for a 2-sided test and alpha of 0.05 , we estimated a sample size of 110 per group (total $=220$ ) to detect a 0.17 difference in rate of children with having $>1$ ED repeat asthma visits over the 12-month follow-up. This is based on assumption of difference in repeat ED visit over 12 months, CON: $P_{0}=0.92$ and INT: $P_{1}=$ 0.75 (difference of 17\%) since 100\% of both groups experienced an ED visit in the prior year. This effect size was chosen based on a similar effect noted in children having a repeat asthma ED visit within 1 year (17\%) following an outpatient follow-up visit versus no follow-up in a similar population. ${ }^{35}$ Standard frequencies, means and standard deviations were used to describe socio-demographic and health characteristics of all children and caregivers at baseline and 12 months. Group differences in variable distributions were 
assessed using Chi-square test statistic for categorical variables, and $t$-test and MannWhitney U test for continuous variables. Cox proportional hazards regression (HR) models, measuring the risk or cumulative probability of experiencing >1 ED revisit over the 12 month follow-up, were calculated to highlight factors associated with a repeat ED visits. Unadjusted analyses examined association between select sociodemographic and health characteristics with risk of a repeat ED visit for all participants and for atopic only children $(83 \%)$. Multivariate regression stepped each factor into the model in order of significance based on univariate test results and theoretical influence (child age and allergic sensitization) to determine the combination of factors related to increased hazard of $>1$ ED visits. Effectiveness of the intervention to reduce the hazard of recurrent ED visits further considered potential factor dependencies and retained in final adjusted model the significant interaction effect between intervention and SHS exposure $(P<0.05)$. Analyses were conducted using SPSS Version 22 software. ${ }^{36}$

\section{3 | RESULTS}

\subsection{Baseline sociodemographic and health characteristics by group}

Overall 204/222 children enrolled had complete data at 12 months (92\%) (Figure 1). Children were primarily male (65\%), African American (94\%), and Medicaid insured (94\%) with a mean age of 6.3 (SD 2.7) years. (Table 2) Children were enrolled year-round with most children enrolled during the fall season but season of enrollment differences were nonsignificant (summer: $12 \%$, fall: $37 \%$, winter: $29 \%$, spring: $22 ; P=0.45$ ). Caregivers were primarily the child's biological mother (94\%), high school or higher educated $(81 \%)$, and single $(75 \%)$. Asthma severity was high with most children categorized with moderate $(46 \%)$ or severe persistent $(29 \%)$ asthma. Over two-thirds $(68 \%)$ of children had one or more asthma ED visits over the 3 months prior to enrollment. Allergic sensitization was prominent with $83 \%$ of children testing positive to one or more allergens with the most common positive allergens as dog (60\%), cat (59\%), mouse (54\%), oak tree $(45 \%)$, timothy grass $(45 \%)$, cockroach $(41 \%)$, and house dust mite $(41 \%)$. Indoor exposure to allergens was high; mouse (47\%), cockroach (32\%), cats (26\%), and dog (19\%) and over half (56\%) of children tested positive for SHS exposure (cotinine $>1.0 \mathrm{ng} / \mathrm{mL}$ ). Despite the high allergen sensitization, few children received asthma specialty care (allergist or pulmonologist) at baseline and/or 12 months (Baseline: 20\%; 12 months: 27\%).

Medication use was primarily non-guideline based. Median controller fills over 12 months prior to enrollment was low at 2.0 fills/12 months (Interquartile Range, IQR: 0-7 fills). Moreover, SABA fills were high with over one-third (39\%) having four or more fills/12 months. AMR values were low (AMR <0.50: 45\%) indicating poor relative controller medication use. Over one-third $(36 \%)$ of care-givers reported being worried about side effects of asthma medications. There were no group differences for any child and caregiver sociodemographic or child health characteristics at baseline.

\subsection{Asthma outcomes at 12 months by group status}

INT children had significantly lower median controller medication fills than CON children (INT: 2.5, CON: 4.0 fills; $P=0.02$ ) but no difference was noted between SABA fills or 
AMR >0.5 (Table 3). There was no difference in symptom free nights over past month between groups, yet a trend was noted in fewer symptom free days over past 2 weeks (INT: 7.8 [SD 5.7], CON: 9.2 [SD 5.1] days; $P=0.06$ ). No other factors were significantly modified by the intervention including cotinine levels, exposure to mouse or cockroach, or caregiver worry about medication side effects.

\subsection{Predictors of repeat ED visits defined as >1 visits over the 12-month follow-up (Unadjusted)}

As seen in Table 4, the only factors associated with any repeat ED asthma visit for all participants were increased number of controller medication fills and having 4 or more SABA fills over the 12-months prior to study enrollment. Regarding controller medication fills, for every increase in number of controller medication fills there was a $4 \%$ increase in the risk of having a repeat ED visit over the 12-month follow-up. Further, children with 4 or more SABA fills had a $58 \%$ increased risk of repeat ED visits for all children. In the unadjusted analysis, no other significant associations were noted between groups for all participants. When limiting the analysis to atopic children, defined as $>1$ positive allergen sensitization, similar trends were noted to result of all participants except for the categorization of children with severe asthma that was significantly associated with increased risk of $>1$ ED visit over the follow-up. Number of positive sensitization tests did not modify the group effect in the hazard ratio (HR) of $>1$ ED visit (interaction effect, $P=$ 0.38 ) nor did having 6 or more positive sensitization tests (interaction effect, $P=0.31$ ).

\subsection{Multivariate model for predictors of increased hazard of $>1$ ED visit over follow-up}

As seen in the final multivariate model for predicting the hazard of a repeat ED visit over the 12 month follow-up (Table 5), children residing in homes with no SHS exposure and in the CON group had an $85 \%$ increased risk, atopic children had a 2.6 times greater risk and younger children and those with an AMR $>0.50$ had an increased risk for a repeat ED visit over the 12 month study period. A borderline significant increase in hazard of repeat ED visits was noted with $>4$ SABA fills. (Table 5). No group differences were noted for risk of repeat asthma ED visits in children with positive SHS exposure. For atopic participants only, similar results were noted with CON participants residing in homes with no SHS exposure had twice the risk of repeat asthma ED visits, and younger children and those with AMR $>0.50$ had increased risk for repeat asthma ED visits.

Time to first repeat ED visit did not differ by group status for children with positive SHS exposure. Among children without SHS exposure, the median time to first recurrent ED visit differed by group (CON: 195; INT: $>365$ days) after adjusting for child age, allergic sensitization status, SABA fills prior to baseline ( $\geq 4$ vs $<4$ per year), AMR ( $<0.50$ vs $\searrow 0.50$ ) and the interaction between group status and SHS exposure. (Figure 2). At the completion of the 12-month follow-up, the cumulative probability of a repeat ED visit in children without SHS exposure was less than $45 \%$ in the INT children compared to almost $70 \%$ of CON children. 


\section{4 | DISCUSSION}

This study indicates that a home-based targeted EC intervention was not effective in reducing repeat asthma ED visits in children with persistent asthma when compared to a control group who received less intensive EC home based intervention. Effective homebased EC interventions are typically multifaceted, intensive and targeted to individual allergen sensitizations and exposures. ${ }^{8,29}$ While our intervention was multifaceted and targeted positive allergen sensitizations, it was not effective in reducing asthma ED visits in children with positive SHS exposure. Effective asthma home based interventions combined education and environmental remediation management ranging from cockroach bait placement to renovation of homes. ${ }^{27}$ The intensity of the EC component of the intervention was likely insufficient when compared to the prior effective home-based interventions, for example, the Inner-City Asthma Study (ICAS) that provided HEPA air and HEPA vacuum cleaners with ongoing integrated pest management services. ${ }^{21}$ A significant relationship was noted between reduction in allergen levels and symptom days in the intervention group. ${ }^{21}$ However, other home environmental interventions have indicated reduction in allergen levels, but not sufficient to achieve a clinical benefit ${ }^{22,23}$ including decreased symptom days and healthcare utilization.

For example, significant reduction in allergen levels (cat, dog, dust mite, cockroach, mouse) was noted in sensitized and exposed inner-city adults and children with asthma and who were assigned to an intensive home based EC intervention including provision of vacuums and HEPA air-purifiers, encouraging reduction of SHS exposure, and implementing stepwise therapy with free asthma medications. ${ }^{23}$ Yet, no reduction in symptom days/nights or use rescue medication was associated with receiving the intervention. ${ }^{23}$ Further, a 12-month professionally delivered integrated pest management intervention for mouse-sensitized and exposed children with asthma demonstrated no statistically significant differences in symptoms days, ED visits or hospitalizations when compared to a pest management education only intervention. ${ }^{22}$ While our intervention was likely insufficient to reduce indoor mouse and pest exposures, we also targeted SHS exposure in the home and caregiver medication education similar to the Childhood Asthma Prevention Study (CAPS). ${ }^{8}$ Lack of achieving a clinical benefit in several of these home-based environmental interventions may be due: (i) modest allergen reductions insufficient to achieve a clinical improvement ${ }^{37}$; (ii) multiple caregiver challenges (personal health problems, stressors and community violence) resulting in non-adherence to the protocol ${ }^{8}$; and (iii) lack of medical management of the child's asthma during the intervention. ${ }^{22}$ Rather, baseline severe asthma was significantly associated with risk of one or more ED visits over the follow-up in our study population, supporting the view that use of guideline controller medication therapy may be a greater priority in treating inner-city children. ${ }^{37}$

Over half of inner-city children with asthma are exposed to SHS $34,38,39$ and it is known that tobacco smoke is an important contributor to childhood wheezing. ${ }^{40}$ Targeting SHS exposure was major component of our intervention rather than limiting protocol to reduce specific allergen exposures. Although INT caregivers of children with positive SHS exposure received brief motivational interviewing by a trained nurse to reduce SHS exposure, no significant reduction in cotinine exposures was associated with the intervention 
at 12 months. Most likely, the reduction in SHS exposure component of the intervention lacked the capacity required to eliminate all SHS exposure for the child. Avoidance of SHS exposures is a key component of NAEPP and international guidelines for the management of childhood asthma ${ }^{31,41}$ and was a major component of this home-based intervention. However, the brief smoking cessation motivational interviewing component of the intervention was likely insufficient for sustained smoking cessation in households with smokers. Caregiver emotional and financial strains to implement a total home smoking ban may elucidate the complexity in reducing a child's SHS exposure. ${ }^{39,42}$ Caregiver misunderstanding of the ubiquitous penetration of SHS in their home and/or residing in multiunit dwellings may have contributed to the null effect of the intervention. ${ }^{39,43}$ Alternatively, health behavior of parents who smoke may explain the lack of decreasing SHS exposure in this sample of high-risk children. Low child influenza immunization rates and/or routine asthma visits were associated with increased level of parental smoking, ${ }^{44}$ suggesting parental reluctance to attend their child's PCP visits as to avoid tobacco smoking admonishment. ${ }^{45}$ Notably, the home-based intervention was effective in a subgroup of children with no SHS exposure. The intervention targeted positive allergen sensitizations for remediation in the home. Perhaps in children with SHS exposure, the added level of complexity needed to reduce SHS home exposure was too difficult to achieve since over half of the children with allergies also had SHS exposure.

Having one or more positive allergen sensitizations was the overwhelming risk associated with a repeat asthma ED visits. Both the caregiver and PCP may under-appreciate the high incidence of allergen sensitization among inner-city individuals with asthma ${ }^{46}$ and this results in under treatment. Allergic sensitization can result in increased airway inflammation and bronchoconstriction and multiple allergen sensitizations and exposure can result in greater adverse effects than sensitization and exposure to a single allergen. ${ }^{47}$ Without knowledge of potential allergen sensitizations, nasal congestion symptoms may be attributed to viral upper respiratory infection and not treated. Notably, asthma and allergic rhinitis have interrelated inflammatory processes in the upper and lower respiratory tract. ${ }^{48-50}$ Nasal turbinate edema from allergic rhinitis can result in direct inhalation of irritants, allergens, and cold air, all potentially causing bronchoconstriction. ${ }^{48}$ Our data support guideline recommendations that children with persistent asthma or failing current asthma management should receive allergen testing. ${ }^{31,51-53}$ Over $80 \%$ of children in our study had positive allergic sensitization, yet, less than one out five children received specialty asthma care. Serologic or skin prick allergy testing in children with asthma is not routinely performed in primary care visits, ${ }^{51}$ yet, these tests can inform step-wise therapy, particularly in children who may have high allergen exposures and/or poorly controlled asthma. 53

Younger child age was associated with increased asthma ED visits. This may be due to early lung function deficits associated with persistent asthma symptoms in children with poorly controlled asthma resulting in a fragile airway leading to bronchospasm and airway inflammation. ${ }^{54,55}$ Further, children attending daycare may experience a complex interaction between viruses and allergen sensitization in early life resulting in persistent asthma symptoms. ${ }^{55}$ Otherwise, increased health-seeking behavior in caregivers of children with new onset asthma or caregivers with fewer resources to manage asthma in the home can lead to use of the ED for asthma management rather than manage symptoms at home. ${ }^{6}$ 
Unadjusted results indicate a 4\% increase in likelihood of an ED visit over the follow-up and in the adjusted multivariable analysis, increased controller medication use (AMR >0.50) zrevisit both suggesting that study children had more severe asthma.

\subsection{Strengths and weaknesses of this study}

Use of biomarkers to confirm SHS exposure and allergen sensitization to specific allergens and the high participant retention rate (92\%) over 12 months are major strengths of this study. Nevertheless, there are several limitations associated with this study. The generalizability of the results is limited in that we purposely recruited children with more uncontrolled asthma and frequent asthma ED visits in order to maximize our ability to detect a difference in asthma symptoms and recurrent asthma ED visits with the intervention program. Thus, our sample of inner-city children may represent a higher percentage of very poorly controlled asthma patients than the general pediatric asthma population. Because medical management was not a component of the intervention protocol, our results may not be generalizable to other community care settings. ${ }^{22}$ The rate of enrollment in the ED; only $51 \%$ of eligible participants, further limits the generalizability but reflects the challenge to enrolling children with asthma into an environmental control intervention during an acute care visit when caregivers are highly stressed. By study design and ethical considerations, our control group received asthma education and support in the home as an attention control group and not usual asthma care. This may have resulted in a significant conservative bias in our analyses, since control group caregivers may have benefited from the receipt of asthma education and increased self-management support for their child's asthma noted in prior home-visiting programs. ${ }^{56}$

\section{5 | CONCLUSIONS}

In conclusion, a home based EC intervention was not associated with a reduction in asthma repeat ED visits among inner-city children with persistent asthma. However, in a subset of children without SHS exposure, the median time to recurrent ED visit was significantly shorter in CON versus INT children. Achieving well-controlled asthma in high-risk young children requires knowledge of allergen sensitization and SHS exposure and a sustainable home-based EC program with ongoing intensive integrated pest management and strategies to reduce SHS exposure.

\section{ACKNOWLEDGMENTS}

The study was funded by The National Institute of Nursing Research, NIH (NR013486). We thank Betty Johnson, RN, and Peter Lukk, RN for their work delivering the interventions, Mary Gates, and Amanda Manning for their work on data collection for this project. This publication was made possible by the Johns Hopkins Institute for Clinical and Translational Research (ICTR) that is funded in part by Grant Number UL1 TR 000424-06 from the National Center for Advancing Translational Sciences (NCATS) a component of the National Institutes of Health (NIH), and NIH Roadmap for Medical Research.

Funding information

National Institute of Nursing Research, NIH, Grant number: NR013486 


\section{REFERENCES}

1. Centers for Disease Control and Prevention. Asthma most recent data. https://www.cdc.gov/asthma/ most_recent_data.htm. (Accessed October 10, 2017).

2. Centers for Disease Control and Prevention. Asthma's Impact on the Nation. https://www.cdc.gov/ asthma/impacts_nation/asthmafactsheet.pdf (Accessed October 10, 2017).

3. Oraka E, Iqbal S, Flanders WD, Brinker K, Garbe P. Racial and ethnic disparities in current asthma and emergency department visits: findings form the National Health Interview Survey: 2001-2010. J Asthma. 2013;50:488-496. [PubMed: 23544662]

4. Mancuso CA, Peterson MG, Gaeta TJ, et al. A randomized controlled trial of self-management education for asthma patients in the emergency department. Ann Emerg Med. 2011;57:603-612. [PubMed: 21227538]

5. Franklin JM, Grunwell JR, Bruce AC, Smith RC, Fitzpatrick AM. Predictors of emergency department use in children with persistent asthma in metropolitan Atlanta, Georgia. Ann Allergy Asthma Immunol. 2017;119:129-136. [PubMed: 28479192]

6. Walsh-Kelly CM, Kelly KJ, Drendel AL, Grabowski L, Kuhn EM. Emergency department revisits for pediatric acute asthma exacerbations. Association of factors identified in an Emergency Department asthma tracking system. Pediatr Emerg Care. 2008;24:505-510. [PubMed: 18645538]

7. Topal E, Gucenmez OA, Harmanci K, Arga M, Derinoz O, Turktas I. Potential predictors of relapse after treatment of asthma exacerbations in children. Ann Allergy Asthma Immunol. 2014;112:361364. [PubMed: 24583137]

8. Klinnert MD, Liu AH, Pearson MR, Ellison MC, Budhiraja N, Robinson Jl. Short-term impact of a randomized multifaceted intervention for wheezing infants in low-income families. Arch Pediatr Adolesc Med. 2005;159:75-82. [PubMed: 15630062]

9. Bellin MH, Collins KS, Osteen P, et al. Characterization of stress in low-income, inner city mothers of children with poorly controlled asthma. J Urban Health. 2017;94:814-823. [PubMed: 28560612]

10. Chipps BE, Corren J, Israel E, et al. Asthma Yardstick: practical recommendations for a sustained step-up in asthma therapy for poorly controlled asthma. Ann Allergy Asthma Immunol. 2017;118:133-142. [PubMed: 28153079]

11. Butz AM, Ogborn J, Mudd S, et al. Factors associated with high short-acting $\beta 2$-agonist use in urban children with asthma. Ann Allergy Asthma Immunol. 2015;114:385-392. [PubMed: 25840499]

12. Gerald LB, Gerald JK, Gibson L, Patel K, Zhang S, McClure LA. Changes in environmental tobacco smoke exposure and asthma morbidity among urban schoolchildren. Chest 2009;135:911916. [PubMed: 19017893]

13. Arroyave WD, Rabito FA, Carlson JC. The Relationship between a specific IgE level and asthma outcomes: results from the 2005-2006 National Health and Nutrition Examination Survey. J Allergy Clin Immunol Pract. 2013;1:501-508. [PubMed: 24565622]

14. Sheehan WJ, Rangsithienchai PA, Wood RA, et al. Pest and allergen exposure and abatement in inner-city asthma: a work group report of the American Academy of Allergy, Asthma \& Immunology Indoor Allergy/Air Pollution Committee. J Allergy Clin Immunol. 2010;125: 575581. [PubMed: 20226293]

15. Fredrickson DD, Molgaard CA, Dismuke SE, Schukman JS, Walling A. Understanding frequent emergency room use by Medicaid-insured children with asthma: a combined quantitative and qualitative study. J Am Board Fam Pract. 2004;17:96-100. [PubMed: 15082667]

16. Pai S, Mancuso CA, Loganathan R, Boutin-Foster C, Basir R, Kanna B. Characteristics of asthmatic patients with and without repeat emergency department visits at an inner city hospital. $\mathrm{J}$ Asthma. 2014;51:627-632. [PubMed: 24588683]

17. Stevens MM, Scribano PV, Gorelick MH. Screening for poor short-term outcome in acute pediatric asthma. Ann Allergy Asthma Immunol. 2007;98:432-439. [PubMed: 17521027]

18. Baltrus P, Xu J, Immergluck L, Gaglioti A, Adesokan A, Rust G. Individual and county level predictors of asthma related emergency department visits among children on Medicaid: a multilevel approach. J Asthma. 2017;54:53-61. [PubMed: 27285734] 
19. Hammer SC, Robroeks CM, van Rij C, et al. Actual asthma control in a paediatric outpatient clinic population: do patients perceive their actual level of control? Pediatr Allergy Immunol. 2008;19:626-633. [PubMed: 18221469]

20. Otsuki M, Eakin MN, Arceneaux LL, Rand CS, Butz AM, Riekert KA. Prospective relationship between maternal depressive symptoms and asthma morbidity among inner-city African American children. J Pediatr Psychol. 2010;35:758-767. [PubMed: 19850709]

21. Morgan WJ, Crain EF, Gruchalla RS, et al. Results of a Home-Based environmental intervention among urban children with asthma. N Engl J Med. 2004;351:1068-1080. [PubMed: 15356304]

22. Matsui EC, Perzanowski M, Peng RD, et al. Effect of an integrated pest management intervention on asthma symptoms among mouse-sensitized children and adolescents with asthma: a randomized clinical trial. JAMA. 2017;317:1027-1036. [PubMed: 28264080]

23. DiMango E, Serebrisky D, Narula S, et al. Individualized household allergen intervention lowers allergen levels but not asthma medication use: a randomized controlled trial. J Allergy Clin Immunol Pract. 2016; 4:671-679. [PubMed: 27025297]

24. Rosenstreich DL, Eggleston P, Kattan M, et al. The role of cockroach allergy and exposure to cockroach allergen in causing morbidity among inner-city children with asthma. N Eng J Med. 1997;336: 1356-1363.

25. Ahluwalia SK, Peng RD, Breysse PN, et al. Mouse allergen is the major allergen of public health relevance in Baltimore City. J Allergy Clin Immunol. 2013;132:830-835. [PubMed: 23810154]

26. Huss K, Adkinson NF, Jr, Eggleston PA, Dawson C, Van Natta ML, Hamilton RG. House dust mite and cockroach exposure are strong risk factors or positive allergy skin test responses in the Childhood Asthma Management Program. J Allergy Clin Immunol. 2001;107:48-54. [PubMed: 11149990]

27. Giese J Evidence-based pediatric asthma intervention and outcome measures in a healthy homes program: an integrated review. J Asthma. 2018;1-12. 10.1080/02770903.2018.1472279. [Epub ahead of print]

28. Welsh EJ, Hasan M, Li P. Home-based educational interventions for children with asthma. Cochrane Database Syst Rev. 2011;10: CD009469.

29. Gold DR, Adamkiewicz G, Arshad SH, et al. NIAID, NIEHS, NHLBI and MCAN Workshop Report: the indoor environment and childhood asthma-implications for home environmental intervention in asthma prevention and management. J Allergy Clin Immunol. 2017;140:933-949. [PubMed: 28502823]

30. Harris PA, Taylor R, Thielke R, Payne J, Gonzalez N, Conde JG. Research electronic data capture (REDCap) - A metadata-driven methodology and workflow process for providing translational research informatics support. J Biomed Inform. 2009;42:377-381. [PubMed: 18929686]

31. U.S. Department of Health and Human Services. National Asthma Education Prevention Program Expert Panel Report 3 (EPR3): Guidelines for the Diagnosis and Management of Asthma. NIH Publication 07-4051. Washington DC: US Department of Health and Human Services; 2007.

32. Schatz M, Zeiger S, Vollmer WM, et al. The controller-to total asthma medication ratio is associated with patient centered as well as utilization outcomes. Chest. 2006;130:43-50. [PubMed: 16840381]

33. Juniper EF, Guyatt GH, Feeny DH, Ferrie PJ, Griffith LE, Townsend M. Measuring quality of life in the parents of children with asthma. Qual Life Res. 1996;5:27-34. [PubMed: 8901364]

34. Kumar R, Curtis LM, Khiani S. A community-based study of tobacco smoke exposure among inner-city children with asthma in Chicago. J Allergy Clin Immunol. 2008;122:754-759. [PubMed: 19014767]

35. Cabana MD, Bruckman D, Bratton SL, Kemper AR, Clark NM. Association between outpatient follow-up and pediatric emergency department asthma visits. J Asthma. 2003;40:741-749. [PubMed: 14626330]

36. IBM Corp. Released 2013 IBM SPSS Statistics for Windows, Version 22.0. Armonk, NY: IBM Corp.

37. Eggleston PA. Can we clear the air? J Allergy Clin Immunol Pract. 2016; 4:680-681. [PubMed: 27393779] 
38. McCarville M, Sohn M, Oh E, Weiss K, Gupta R. Environmental tobacco smoked and asthma exacerbations and severity: differences between measured and reported exposure. Arch Dis Child. 2013;98: 510-514. [PubMed: 23606711]

39. Butz AM, Breysse P, Rand C, et al. Household smoking behavior: effects on indoor air quality and health of urban children with asthma. Matern Child Health J. 2011;15:460-468. [PubMed: 20401688]

40. Gergen PJ, Fowler JA, Maurer KR, Davis WW, Overpeck MD. The burden of environmental tobacco smoke exposure ion the respiratory health of children 2 months through 5 years of age in the United States: third national health and nutrition examination survey. Pediatrics. 1998;101:E8.

41. Global Initiative for Asthma (GINA). Global Strategy for Asthma Management and Prevention, 2017 http://ginasthma.org/2017-gina-report-global-strategy-for-asthma-management-andprevention/ accessed October 30, 2017.

42. Chalton M, Diemet L, Cohen JE, et al. Estimating the number of quit attempts it takes to quit smoking successfully in a longitudinal cohort of smokers. BMJ Open. 2016;6:e011045.

43. Bellin MH, Land C, Newsome A, et al. Caregiver perception of asthma management of children in the context of poverty. J Asthma. 2017;54:162-172. [PubMed: 27304455]

44. Pyle RC, Divekar R, May SM, et al. Asthma-associated comorbidities in children with and without secondhand smoke exposure. Ann Allergy Asthma Immunol. 2015;115:205-210. [PubMed: 26208757]

45. Crombie IK, Wright A, Irvine L, Clark RA, Slane PW. Does passive smoking increase the frequency of health service contacts in children with asthma? Thorax. 2001;56:9-12. [PubMed: 11120897]

46. Gruchalla RS, Pongracic J, Plaut M, et al. Inner City Asthma Study: relationship among sensitivity, allergen exposure, and asthma morbidity. J Allergy Clin Immunol. 2005;115:478-485. [PubMed: 15753892]

47. Matsui EC. Environmental exposures and asthma morbidity in children living in urban neighborhoods. Allergy. 2014;69:553-558. [PubMed: 24697316]

48. Togias A. Rhinitis and asthma: evidence for respiratory system integration. J Allergy Clin Immunol 2003;111:1171-1183. [PubMed: 12789212]

49. Bousquet J, Gaugris S, Sazonov-Kocevar V, et al. Increased risk of asthma attacks and emergency room visits among asthma patients with allergic rhinitis: a subgroup analysis of the improving asthma control trial. Clin Exp Allergy. 2005;35:723-727. [PubMed: 15969661]

50. Valovirta E, Pawankar R. Survey on the impact of comorbid allergic rhinitis in patients with asthma. BMC Pulm Med. 2006;6:1-10. [PubMed: 16412223]

51. Erickson S, Tolstykh I, Selby JV, Mendoza G, Iribarren C, Eisner MD. The impact of allergy and pulmonary specialist care on emergency asthma utilization in a large managed care organization. Health Serv Res. 2005;40:1443-1465. [PubMed: 16174142]

52. Sheares BJ, Mellins RB, Dimango E, et al. Do patients of subspecialist physicians benefit from written asthma action plans? Am J Resp Crit Care Med. 2015;191:1374-1383. [PubMed: 25867075]

53. Anderson WC, 3rd, Szefler SJ. Controlling the risk domain in pediatric asthma through personalized care. Semin Respir Crit Care Med. 2018; 39:36-44. [PubMed: 29427984]

54. To T, Dick P, Feldman W, Hernandez R. A cohort study on childhood asthma admission and readmission. Pediatrics. 1996;98:191-195. [PubMed: 8692616]

55. Martinez FD. New insights into the natural history of asthma: primary prevention on the horizon. $\mathrm{J}$ Allergy Clin Immunol. 2011;128:939-945. [PubMed: 22036094]

56. Sawyer MG, Barnes J, Frost L. Nurse perceptions of family home-visiting programmes in Australia and England. J Paediatr Child Health. 2013;49:369-374. [PubMed: 23573991] 


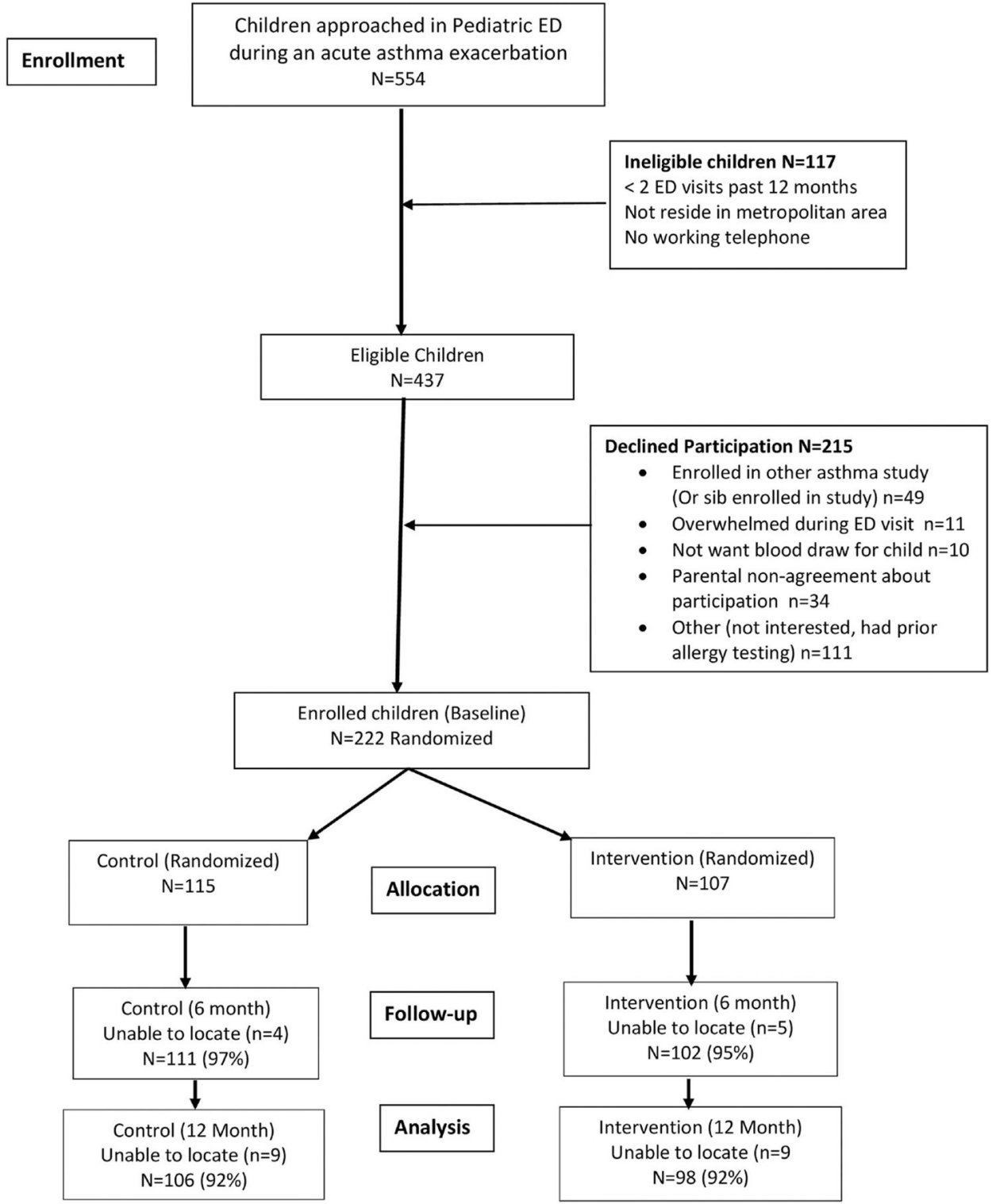

FIGURE 1.

Recruitment, Randomization and Retention of Participants Diagram 


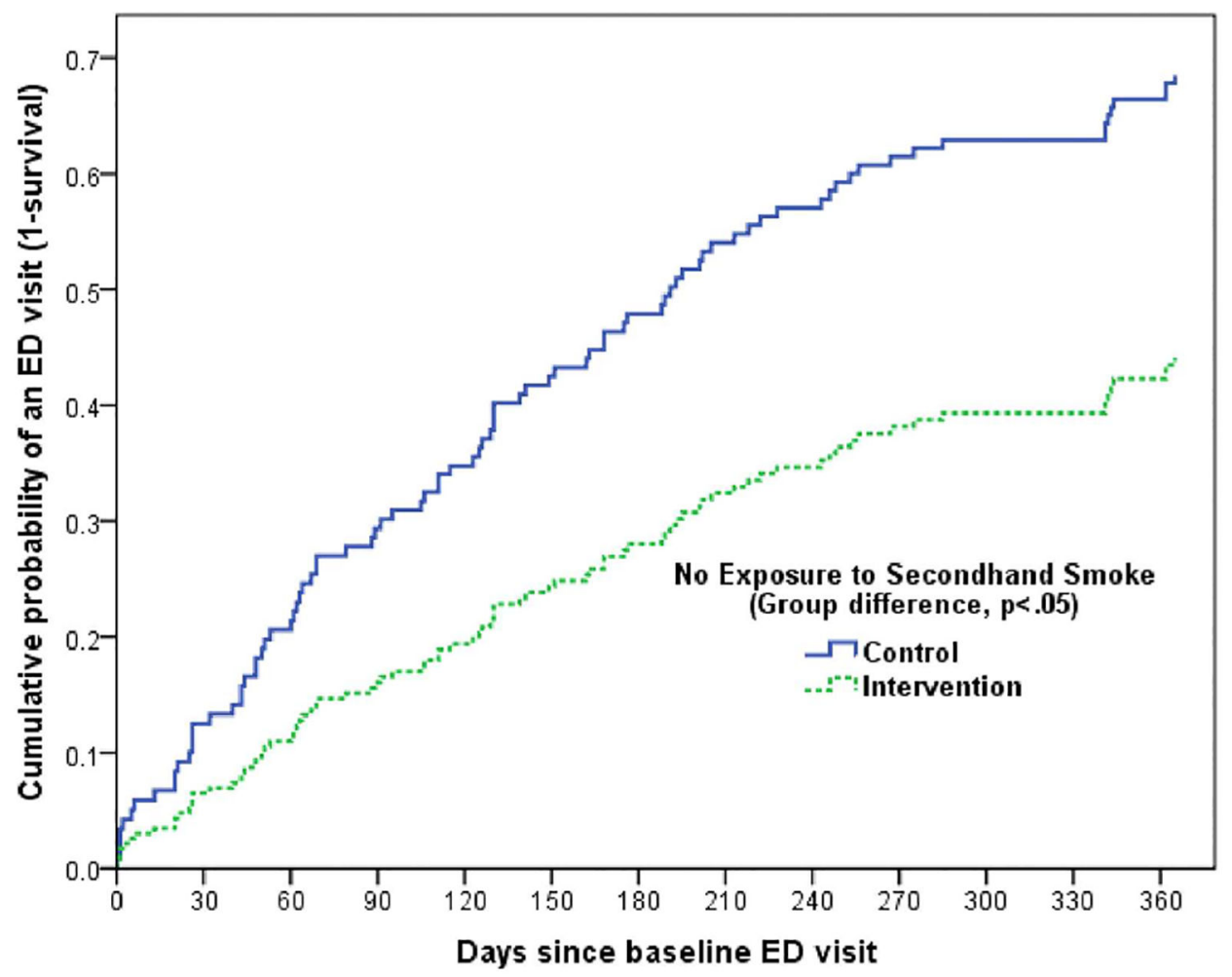

FIGURE 2.

Cumulative probability for a child participant to have a repeat ED visit with each additional day following the baseline ED enrollment visit (repeat ED visit over follow-up) presented in children residing in homes with NO SHS exposure.a 

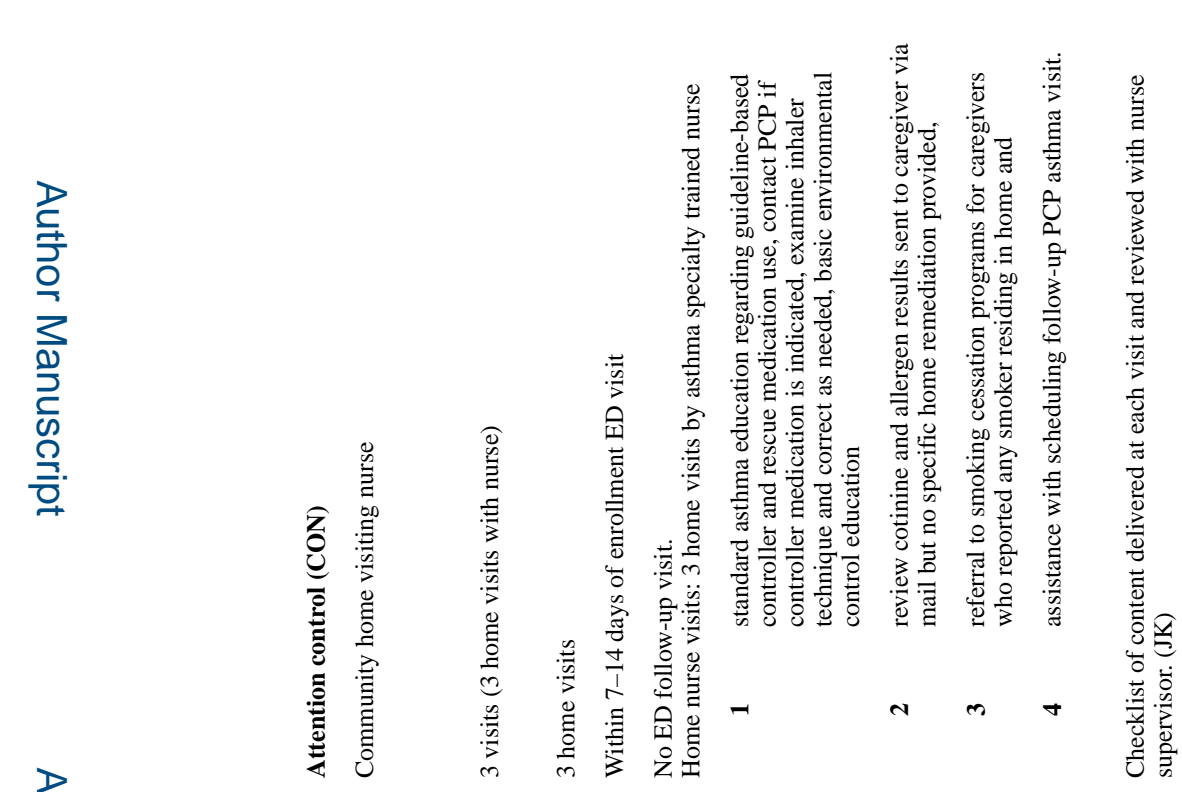

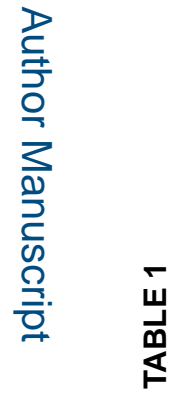

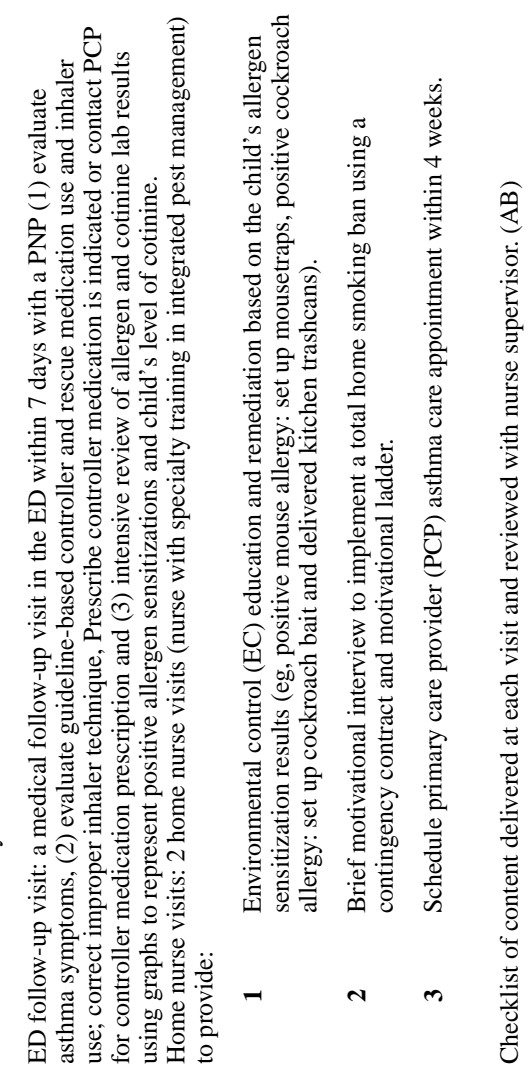

? 

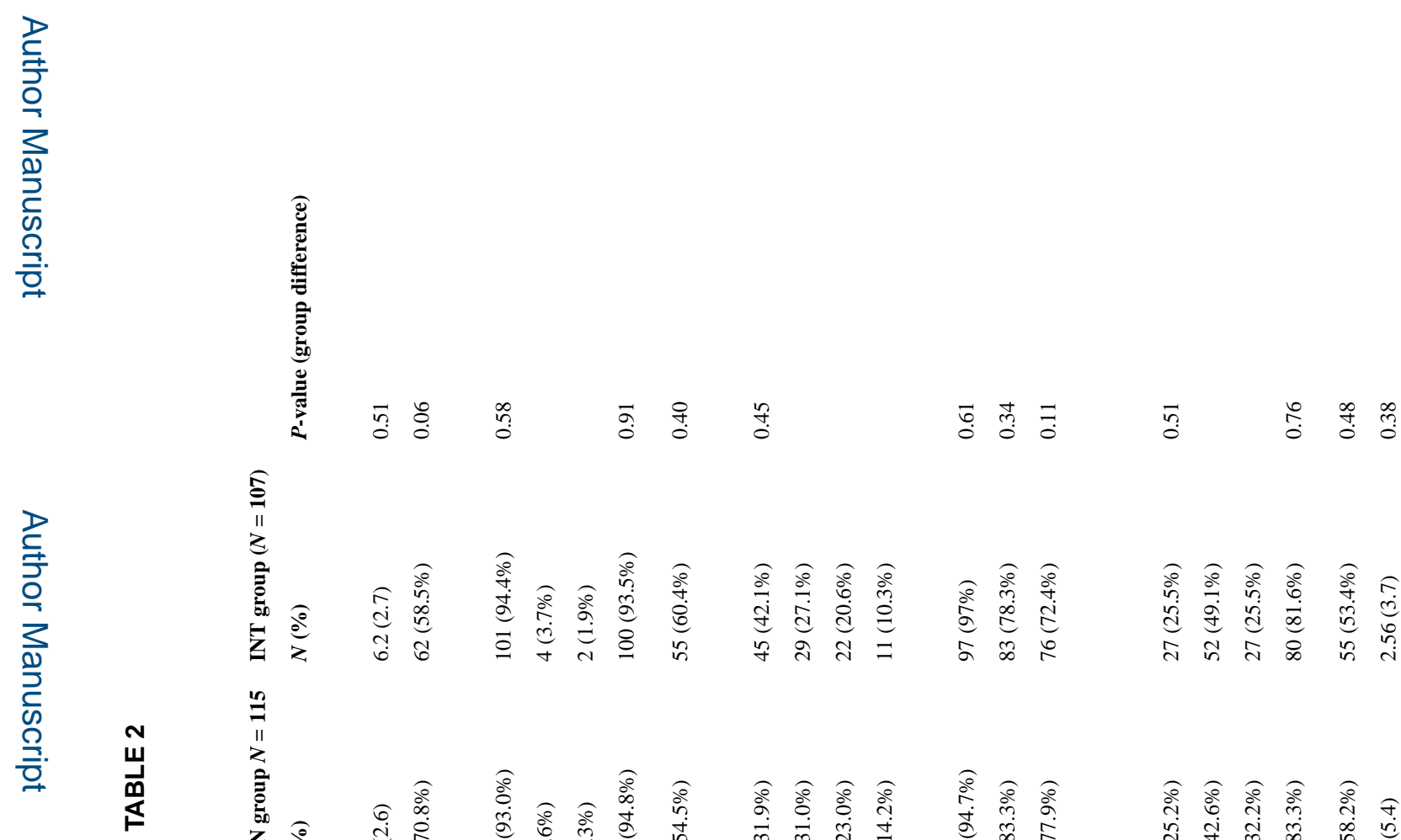

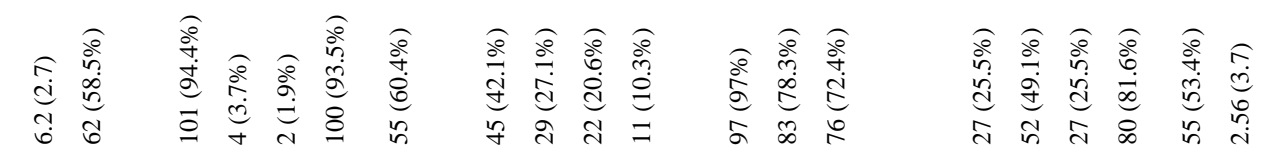

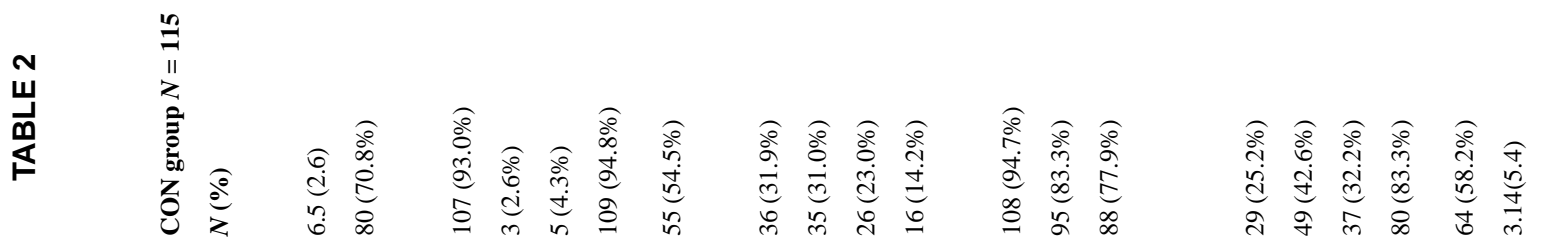

要

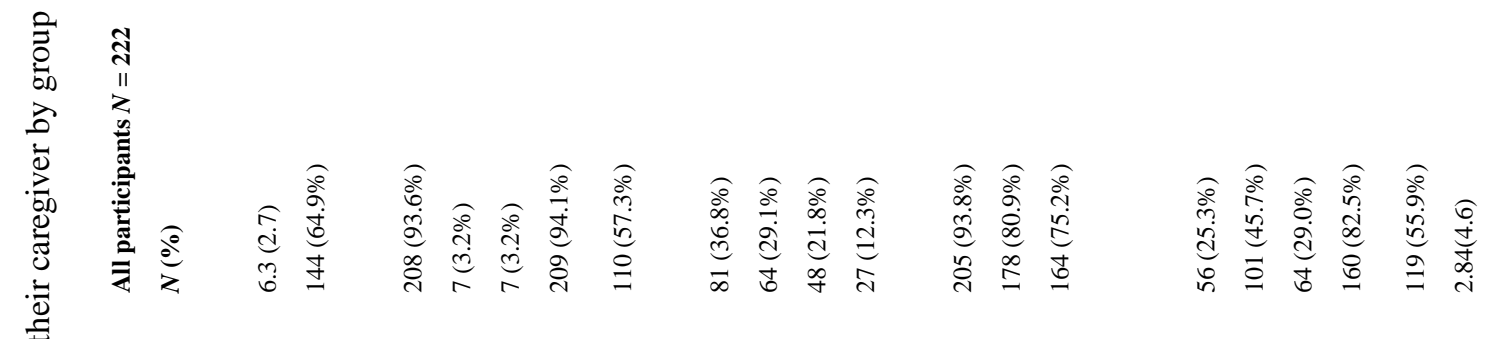




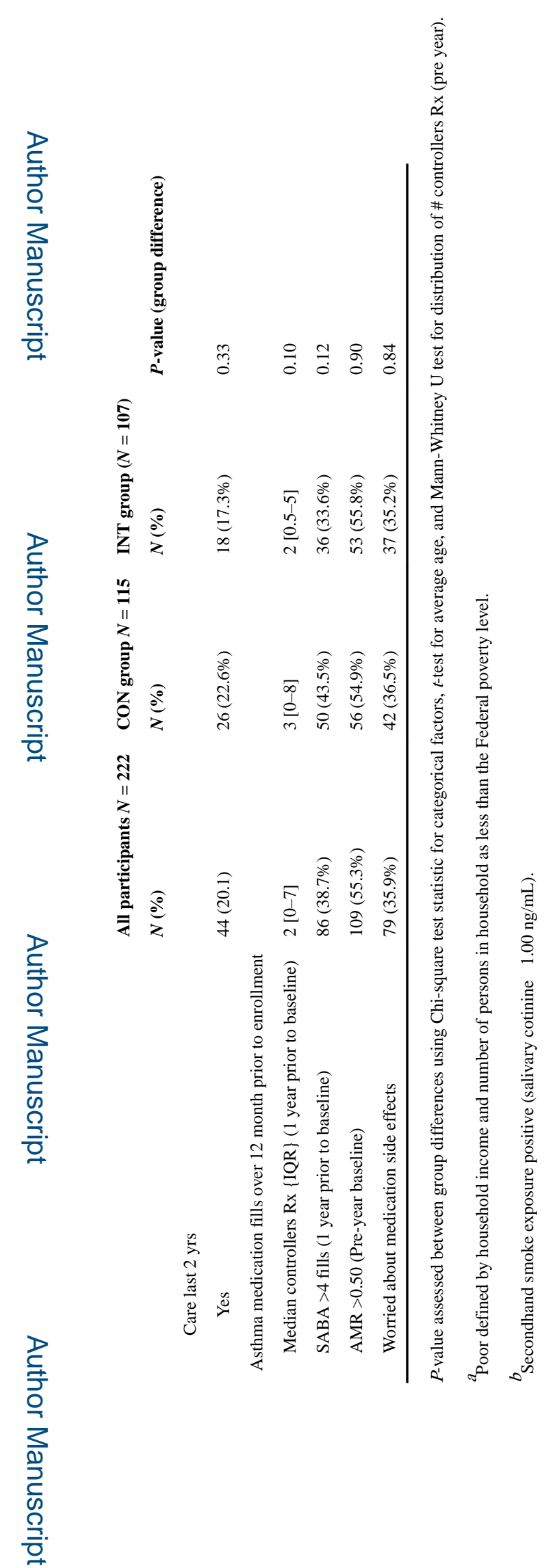

Pediatr Pulmonol. Author manuscript; available in PMC 2020 March 01. 

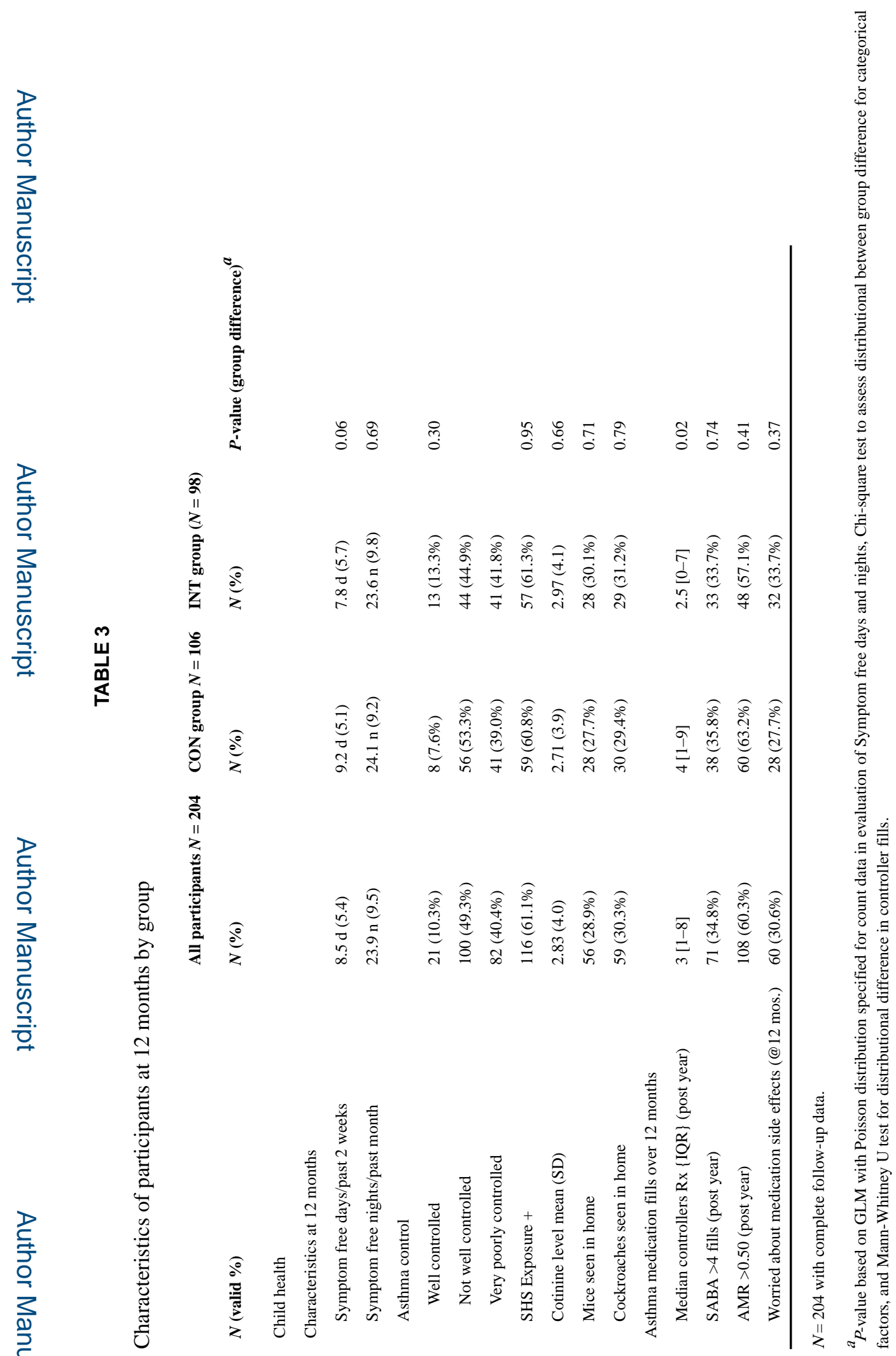

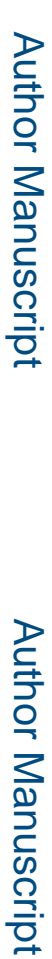

Pediatr Pulmonol. Author manuscript; available in PMC 2020 March 01. 


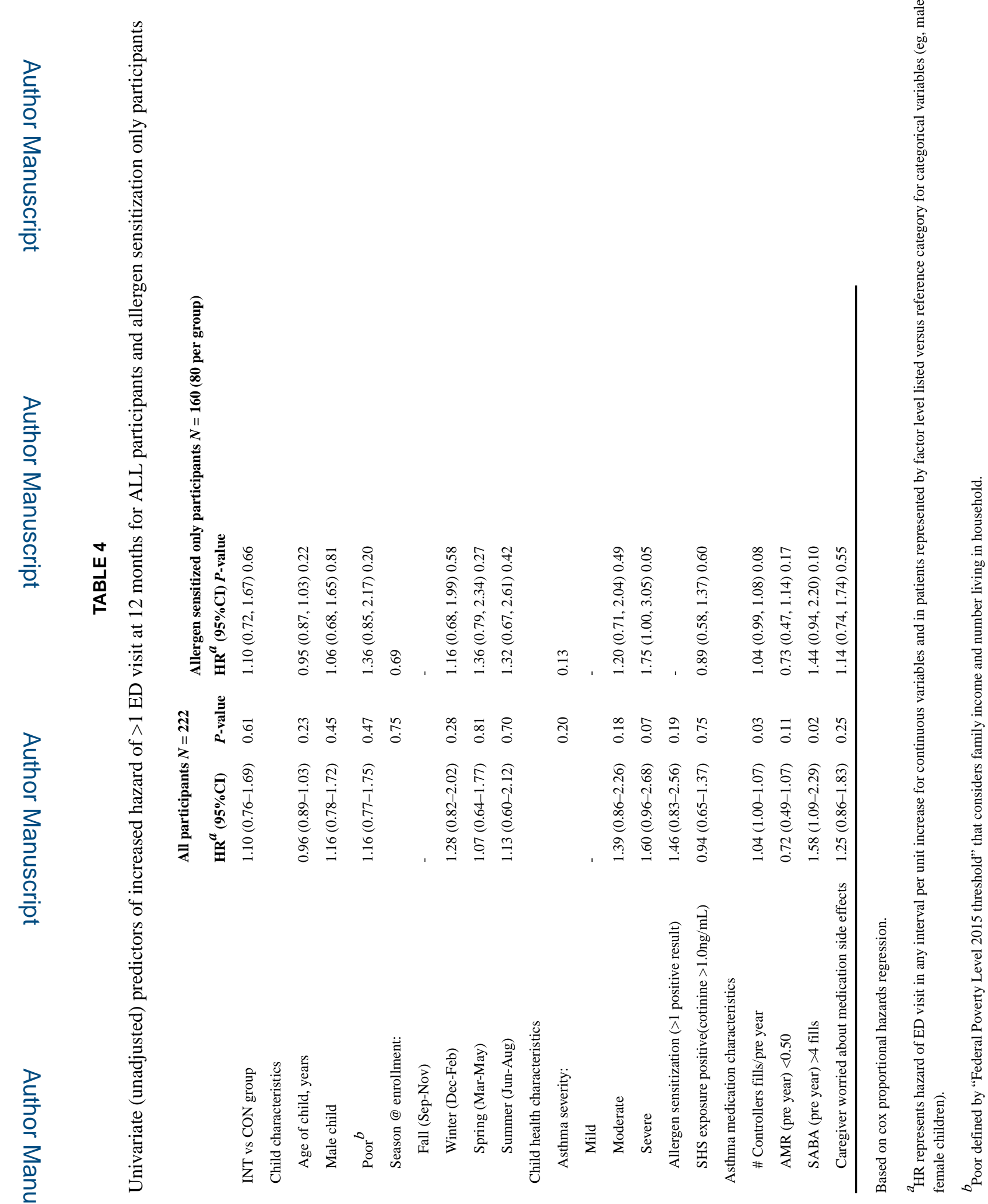

Pediatr Pulmonol. Author manuscript; available in PMC 2020 March 01. 


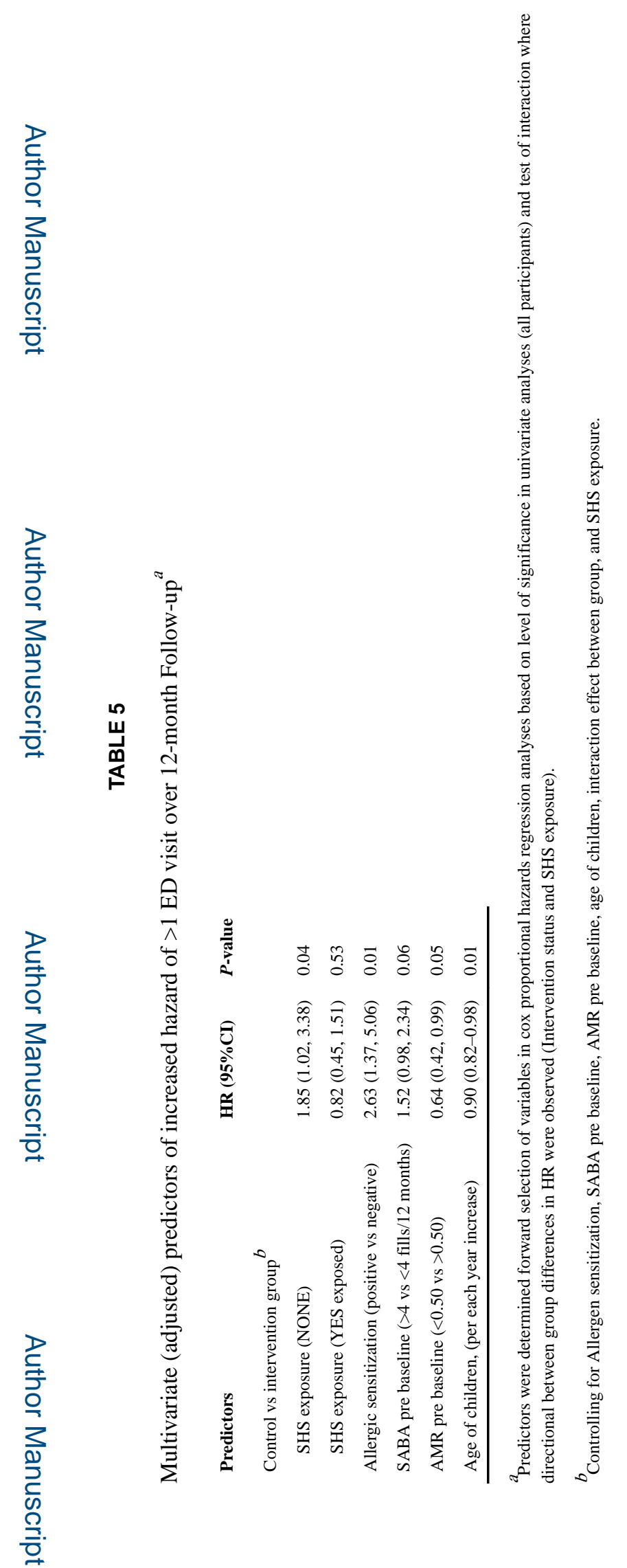

Pediatr Pulmonol. Author manuscript; available in PMC 2020 March 01. 